\title{
Inspection of Defect-Induced Mode Coupling in Hollow-Core Photonic Bandgap Fibers Using Time-of-Flight
}

\author{
Nicholas H. L. Wong*, S. Reza Sandoghchi, Yongmin Jung, Tom Bradley, Natalie V. Wheeler, N. K. Baddela, \\ John R. Hayes, F. Poletti, Marco N. Petrovich, Shaif-Ul Alam, Periklis Petropoulos, and David J. Richardson \\ Optoelectronics Research Centre, University of Southampton, Southampton SO17 1BJ, UK \\ *nhlw1g12@orc.soton.ac.uk
}

\begin{abstract}
We analyze defect-induced mode coupling in a hollow-core photonic bandgap fiber using time-of-flight, and show its utility in complementing optical time-domain reflectometry. OCIS codes: (060.2270) Fiber characterization; (060.2330) Fiber optics communications; (060.4005) Microstructured fibers.
\end{abstract}

\section{Introduction}

Hollow-core photonic bandgap fibers (HC-PBGFs) are receiving considerable interest for both high-capacity singlemode and multimode data transmission using mode-division multiplexing, notably in the face of a potential future capacity crunch [1]. Although current state-of-the-art fabrication techniques are generally robust, reliable, and reproducible in their performance, it is still possible that some fibers produced may contain longitudinal defects, particularly over multi-km lengths, and understanding the origin and impact of these will be critical in fabricating long lengths of fiber [2]. Such defects can occur in a variety of forms including structural irregularity, microfractures, and contamination, and can be anticipated to lead to increased transmission loss, undesirable sources of mode coupling, and/or possibly mode dependent loss. These are problematic especially when long length $(>\mathrm{km})$ scales are considered, making detection and ultimately elimination of defects a pertinent challenge.

The prevailing method for locating defects along conventional fibers is optical time domain reflectometry (OTDR), though there are questions about its accuracy in multimode fibers [3]. OTDR also does not provide much information about how the defects affect individual mode propagation and coupling, and fibers containing defects, which may not necessarily exhibit strong mode coupling and may still be usable for transmission, are often disregarded.

The time-of-flight (ToF) method is well-suited to study mode coupling effects. Here, we demonstrate an extension to its utility by detecting longitudinal fiber defects and enhancing OTDR information.

\section{Setup and Method}

Fig. 1(a) shows the experimental setup for ToF, which is similar to that in [4]. A passively mode-locked pulsed fiber laser (PFL) operating at a repetition rate of $20 \mathrm{MHz}$, followed by an optical bandpass filter, produces Gaussian-shaped few-picosecond pulses at $1550 \mathrm{~nm}$. The pulses are amplified and coupled to a free-space segment including a binary phase plate (PP) to selectively excite higher-order modes (HOMs) and a telecentric imaging system to improve coupling efficiency. The light is coupled to the fiber under test, whose output is fed to a $10 \mathrm{GHz}$ sampling oscilloscope. Modal dispersion separates successive mode peaks and enables them to be individually resolved temporally.

(a)

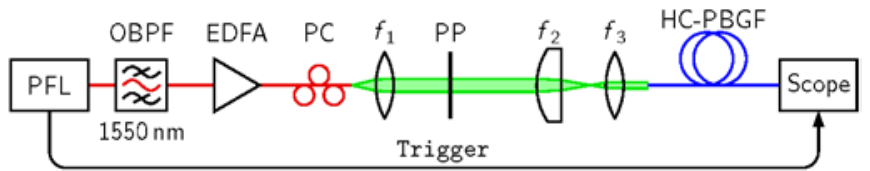

(b)

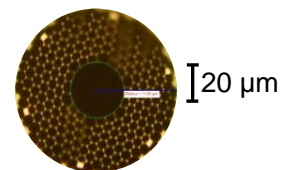

Fig. 1: (a) ToF setup. PFL = Pulsed fiber laser, $\mathrm{OBPF}=$ optical bandpass filter, $\mathrm{EDFA}=$ erbium-doped fiber amplifier, $\mathrm{PC}=$ polarization controller, PP = phase plate. (b) Cross-sectional image of the fiber hollow core and cladding structure.

Shifting the transverse position of the PP selectively launches individual linearly polarized (LP) modes, and transitions from one mode to another can be seen in real-time on the oscilloscope display as corresponding peaks grow or diminish.

\section{Measurements on Hollow-core Photonic Bandgap Fiber}

We first used OTDR to locate the position of defects in an $L=281 \mathrm{~m}$ long 19-cell HC-PBGF known to have several defects. Fig. 3(a) shows the OTDR traces for the selective excitation of $\mathrm{LP}_{01}$ and $\mathrm{LP}_{11}$. Four prominent defects are observed (labelled A, B, C, and D) along the fiber. Note that the relative reflection amplitudes of the peaks are mode dependent and different for either mode excitation, i.e. the biggest reflection defects are $\mathrm{D}$ for $\mathrm{LP}_{01}$ excitation and $\mathrm{B}$ for $\mathrm{LP}_{11}$. As per the concerns raised in [3], we remark that the OTDR traces are not concretely representative of actual loss, and are merely used to identify defects. Fig. $3(\mathrm{~b})$ depicts the ToF trace for $\mathrm{LP}_{01}$ launch, with the $\mathrm{LP}_{01}$ main mode component at $\Delta t_{01}=0 \mathrm{~ns}$, and Fig. 3(c) shows the ToF trace for $\mathrm{LP}_{11}$ launch. The main $\mathrm{LP}_{11}$ mode group is composed 
of individual vector mode peaks which spread out due to the higher sensitivity of $\mathrm{LP}_{11}$ to fiber birefringence. We identify two $L P_{11}$ peaks 1 and 2 with relative delays of $\Delta t_{11,1}=2.6 \mathrm{~ns}$ and $\Delta t_{11,2}=3.5 \mathrm{~ns}$, respectively. The large plateau within the $\mathrm{LP}_{11}$ mode group results from strong cross coupling among the constituent vector modes. Excitation of the much lossier HOMs beyond $\mathrm{LP}_{11}$ produced no discernible $\mathrm{ToF}$ features. The ToF and OTDR traces have been scaled such that the time delay between $\Delta t_{01}$ and $\Delta t_{11,1}$ correspond to the total length of the fiber (black dashed lines). ToF features occurring between these delays are a result of defect-induced mode coupling because coupled mode components arising from defects have net relative delays that are combinations of the main $\mathrm{LP}_{01}$ (faster) and $\mathrm{LP}_{11}$ (slower) mode delays. Defect features in Fig. 3(b) are $C_{01 \rightarrow 11}$ events, where we use the notation $C_{a \rightarrow b}$ to denote coupling from mode $\mathrm{LP}_{a}$ to $\mathrm{LP}_{b}$, whereas features in Fig. 3(c) are $C_{11 \rightarrow 01}$ events. Furthermore, the vector mode spread in $\mathrm{LP}_{11}$ manifests such that the same defect produces multiple ToF peaks. For example, in Fig. 3(b), defect $D$ (appearing as the largest $\mathrm{LP}_{01}$ OTDR feature at $\ell_{\mathrm{D}}=227.7 \mathrm{~m}$ in Fig. 3(a)) causes the $C_{01 \rightarrow 11}$ ToF feature group [D1; D2]. Their delays can be calculated from $\left[\Delta t_{\mathrm{D} 1} ; \Delta t_{\mathrm{D} 2}\right]=\left(1-\ell_{\mathrm{D}} / L\right) \times\left[\Delta t_{11,1} ; \Delta t_{11,2}\right]=\sim[0.5 ; 0.7]$ ns, which matches what we observe on the ToF trace. Similarly, the large OTDR defect $\mathrm{B}$, at $\ell_{\mathrm{B}}=112.5 \mathrm{~m}$ in Fig. 3(a) for $\mathrm{LP}_{11}$ launch, produces the $C_{11 \rightarrow 01}$ ToF feature group [B1; B2] in Fig. 3(c), with $\left[\Delta t_{\mathrm{B} 1} ; \Delta t_{\mathrm{B} 2}\right]=\left(\ell_{\mathrm{B}} / L\right) \times\left[\Delta t_{11,1} ; \Delta t_{11,2}\right]=\sim[1.0 ; 1.4]$ ns, again matching with the ToF trace. Note the difference between using $(1-\ell / L)$ as the scaling factor for the $C_{01} \rightarrow 11$ calculation and $(\ell / L)$ for $C_{11 \rightarrow 01}$. The physical basis for this is that $C_{01 \rightarrow 11}$ and $C_{11 \rightarrow 01}$ events cause slower and faster subsequent propagation, respectively; e.g. $C_{01 \rightarrow 11}$ events occurring near the end of the fiber would cause ToF features at smaller delay deviations from the main $\mathrm{LP}_{01}$ peak, since the coupled mode components would propagate as the slower $\mathrm{LP}_{11}$ over only a short distance, and vice versa. The feature delay spreads (e.g. $\left|\Delta t_{\mathrm{D} 2}-\Delta t_{\mathrm{D} 1}\right|$ and $\left.\left|\Delta t_{\mathrm{B} 2}-\Delta t_{\mathrm{B} 1}\right|\right)$ also scale correctly with these respective factors. The fact that different defects produce ToF features for different mode excitations implies some mode selectivity. Certain defects, e.g. A, may not even produce significant coupling among modes under study, meaning that fibers, even with such defects, may still be transmission-worthy for specific modes.

(a)

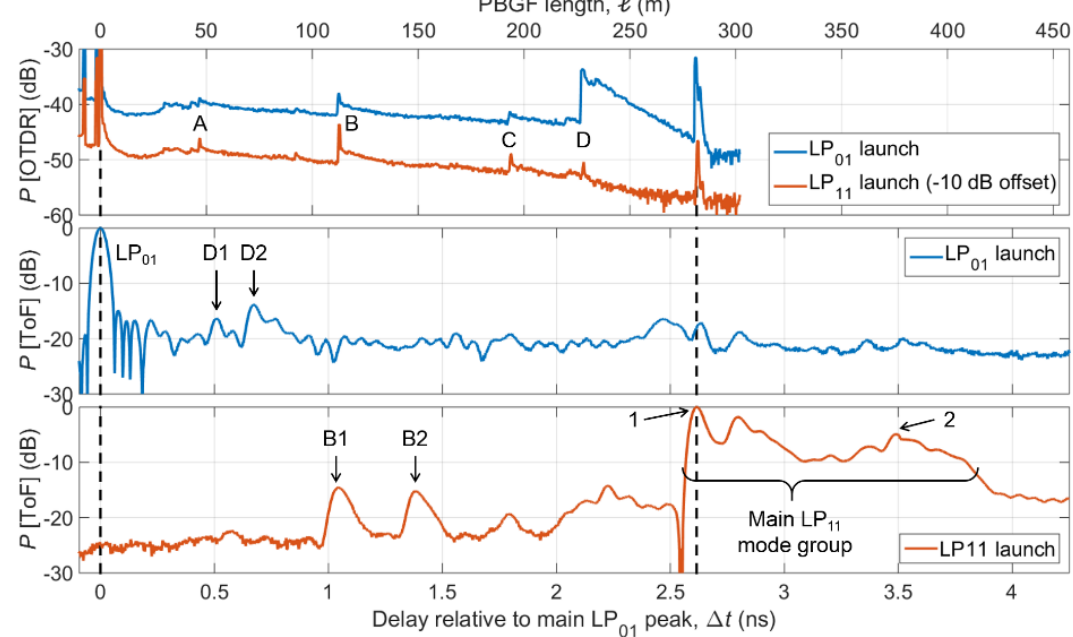

(d)

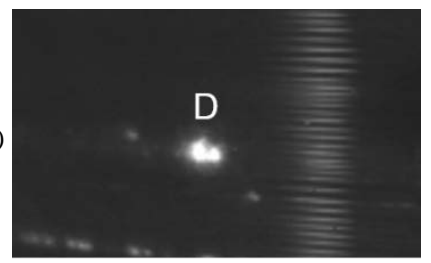

(e)

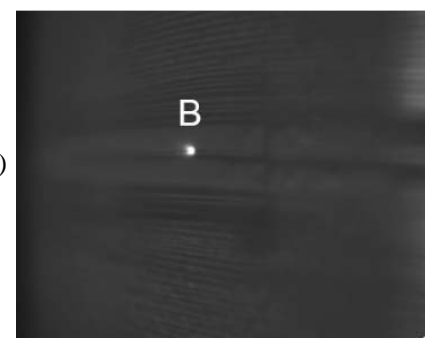

Fig. 2: (a) OTDR and (b-c) ToF traces for selective $\mathrm{LP}_{01}$ and $\mathrm{LP}_{11}$ launches in the 19-cell HC-PBGF under test. $P=$ power. (d-e) Side-scattered light showing defects $\mathrm{D}$ and $\mathrm{B}$, respectively.

There is thus a direct correlation between OTDR defect position and expected ToF feature delay. Figs. 3(d) and 3(e) display bright spots of side-scattered infrared light captured using the method in [2], which correspond to defects $\mathrm{D}$ and $\mathrm{B}$, respectively. We further verified the correspondence between defect position and ToF delay location experimentally by non-destructively forcing manual tactile perturbations at such bright spots and other positions along the fiber and observing the real-time growth of the respective ToF regions.

\section{Conclusions}

Using ToF, we are able to detect longitudinal defects in HC-PBGFs and also the consequential mode coupling behavior. We showed that ToF can complement OTDR by providing more information to determine transmission capability at certain modes. Further work can be done to quantity the amount of coupling from each defect and also determine the physical origin of the mode selectivity.

This work was supported by the European Communities 7th Framework Programme under grant agreement 258033 (MODE-GAP).

\section{References}

[1] E. Desurvire et al., Comptes Rendus Physique 12 (4), 387-416 (2011).

[3] M. Belal et al., IEEE Photon. Technol. Lett. 26 (9), 889-892 (2014).

[2] S. R. Sandoghchi et al., in Proc. OFC 2014, M2F.6 (2014).

[4] F. Poletti et al., Nat. Photon. 7 (4), 279-284 (2013). 